Annales Geophysicae (2002) 20: 1279-1289 (C) European Geophysical Society 2002

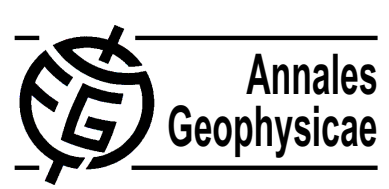

\title{
EISCAT measurements of solar wind velocity and the associated level of interplanetary scintillation
}

\author{
R. A. Fallows, P. J. S. Williams, and A. R. Breen \\ Physics Department, University of Wales, Aberystwyth, SY23 3BZ, Wales, UK \\ Received: 17 October 2001 - Revised: 30 April 2002 - Accepted: 17 May 2002
}

\begin{abstract}
A relative scintillation index can be derived from EISCAT observations of Interplanetary Scintillation (IPS) usually used to study the solar wind velocity. This provides an ideal opportunity to compare reliable measurements of the solar wind velocity derived for a number of points along the line-of-sight with measurements of the overall level of scintillation. By selecting those occasions where either slow- or fast-stream scattering was dominant, it is shown that at distances from the Sun greater than $30 R_{S}$, in both cases the scintillation index fell with increasing distance as a simple power law, typically as $R^{-1.7}$. The level of scintillation for slow-stream scattering is found to be 2.3 times the level for fast-stream scattering.
\end{abstract}

Key words. Interplanetary physics (solar wind plasma)

\section{Introduction}

Interplanetary scintillation (IPS) is the fluctuation of the radio signal from a compact, extra-galactic radio source after the line-of-sight from source to Earth has passed through density fluctuations in the solar wind. IPS has been used to study the solar wind close to the Sun for more than three decades (e.g. Hewish et al., 1964; Dennison and Hewish, 1967).

Phase variations introduced by the density fluctuations scatter the signal and cause the fluctuations in received power. If the phase variations introduced across the wavefront are large ( $\gg 1$ radian), then the scattered waves do not add constructively and the variations in received power are relatively small; this is known as 'strong' scattering. If, however, the phase variations are small ( $\ll 1$ radian) then the scattered waves add constructively to generate much larger fluctuations in received power (e.g. Little and Hewish, 1966; Bourgois, 1969). This is known as 'weak' scattering.

The standard measurement of the level of IPS is $m$, the scintillation index. This is the ratio of the rms variation in

Correspondence to: R. A. Fallows (raf@aber.ac.uk) the strength of the source signal due to IPS to the average strength of the signal.

As the distance from the Sun increases, the average electron density in the solar wind and the rms variation in electron density decrease, so that the phase variation across the wavefront also decreases. It follows that if the line-of-sight passes close to the Sun, then the phase variation is large, strong scattering occurs, and $m$ is low. As the distance of the line-of-sight from the Sun increases, there is a transition from strong to weak scattering, so at first $m$ increases with solar distance until it reaches a peak. At even greater distances from the Sun, only weak scattering occurs and $m$ decreases with solar distance $R$.

Within the weak scattering regime, the scintillation index is directly proportional to the variation in solar wind electron density, which, in turn, is related to the mean electron density. Thus, observations have shown that $m$ is lower at high latitudes, where the solar wind fast-stream above polar coronal holes is less dense, than at low latitudes, where the measurements are associated with the equatorial streamer belt and the much denser slow stream (e.g. Bourgois and Coles, 1992; Manoharan, 1993; Asai et al., 1998).

In addition, the scintillation index is dependent on the structure of the radio source being observed. For an ideal point source, $m$ is expected to peak near unity. However, as the angular diameter of the scintillating source increases, the scintillation level decreases (Briggs, 1961). This effect is especially marked if a compact source is surrounded by an extended component that adds significantly to the overall power of the source, so that the apparent scintillation index is reduced. Measurements of the scintillation index have usually been carried out by large monostatic antennas capable of observing large numbers of sources every day to obtain a two-dimensional image of the distribution of $m$ over a wide range of heliographic latitudes and distances from the Sun (e.g. Gapper et al., 1982). These images can be compared with data from spacecraft and other available sources, but the variation of solar wind parameters along the line-of-sight from source to Earth, such as velocity, could not be derived 
from single observations.

More recently, a method of deriving the solar wind velocity from single-station observations has been developed (Manoharan and Ananthakrishnan, 1990). As scattering power falls off rapidly with distance, $R$, from the Sun $(\alpha$ $R^{-4}$ ), it was assumed that the derived velocities referred to the points of closest approach of the lines-of-sight to the Sun. In reality, however, there are contributions to the total scattering power from along the entire line-of-sight. The development of tomography (Jackson et al., 1998; Kojima et al., 1998; Asai et al., 1998) as a technique for modeling the solar wind in three dimensions has helped to reduce this problem, but this method is reliant on the combination of a large number of observations, which reduces the temporal resolution.

When two or more antennas are available, a suitable distance apart, simultaneous observations of the same scintillating source allow for a cross-correlation analysis of the scintillations to be made and from this the solar wind velocity across the lines-of-sight can be estimated. This technique for measuring solar wind velocity has been used for many years (e.g. Dennison and Hewish, 1967; Vitkevich and Vlasov, 1970). The UHF antennas of EISCAT in northern Scandinavia have been used in this mode since 1990 to make regular measurements of solar wind velocity. In the early EISCAT measurements (Bourgois et al., 1985), it was assumed that the solar wind velocity was constant along the line-of-sight. However, later observations used the maximum antenna spacing (up to $390 \mathrm{~km}$ ) and with the improved velocity resolution this provided it became clear that the crosscorrelation function often showed two distinct peaks, corresponding to separate regions of fast and slow solar wind streams along the line-of-sight (e.g. Breen et al., 1996b). Observations by Ulysses had already demonstrated a clear distinction between fast- and slow-stream regimes in the solar wind (Phillips et al., 1995).

For many years it has been accepted that the fast wind is associated with coronal holes (e.g. Schwenn et al., 1978), which show up as dark regions in white-light images of the corona. Therefore, Carrington maps of coronal white light (e.g. from the High Altitude Observatory) give a clear indication where it is likely that the line-of-sight passes through fast- or slow-stream regions of the solar wind. Subsequent analysis of the EISCAT IPS data was carried out using a twodimensional weak scattering model (Grall, 1995; Klinglesmith, 1997; Massey, 1998), which assumed the presence of two streams, as indicated by linking each part of the line-ofsight to the corresponding part of the Carrington map (Coles, 1996). This analysis not only gave the values of the fast- and slow-stream velocities, but also included the relative contribution of each to the total scattering in a single observation. The analysis procedure is detailed further elsewhere (e.g. Coles, 1996; Breen et al., 1996b; Grall et al., 1996; Breen et al., 1998; Canals et al., 2002).

Use of this revised analysis procedure gave very good agreement between the observed cross-correlation function and the best-fitting model. A generally good agreement is also found when the results from a series of two-station EIS-
CAT observations of solar wind velocity are compared with corresponding single-station observations taken by the Ooty Radio Telescope (ORT) (Moran et al., 2000). The good agreement obtained has given further confidence in the different analysis methods used by EISCAT and ORT to determine solar wind velocity.

As well as determining solar wind velocity, the EISCAT observations can also be used to determine scintillation indices, and this has been done for all observations of IPS made between 1994 and 1999. These are compared with distance from the Sun, but for the present paper only those cases where either a fast- or slow-stream dominates along the whole line-of-sight have been used. The novel factor of these results is that this is the first time that measurements of the scintillation index can be divided between fast- and slowstream scattering regimes with some confidence for each observation.

\section{Scintillation index}

A relative scintillation index can be calculated from EISCAT interplanetary scintillation measurements using parameters that are easily measured or confidently assumed. $I(t)$, the total detected signal ${ }^{1}$ at the output of the receiving system, can be described by:

$$
I(t)=\langle N\rangle+I_{S}+\delta N(t)+M(t) I_{S}
$$

where $\langle N\rangle$ is the average background or system noise; $I_{S}$ is the average signal from the radio source; $\delta N(t)$ is the stochastic variation in the background noise; $M(t)$ is a scintillation factor whose rms value is $m$.

$P_{I}(f)$, the power spectrum of the time-variations of the signal is given by:

$P_{I}(f)=P_{N}(f)+P_{S c}(f)=P_{N}(f)+P_{M}(f) I_{S}^{2}$,

where $P_{N}(f)$ is the power spectrum of the stochastic variation of the background; $P_{S c}(f)$ is the power spectrum of the scintillations; $P_{M}(f)$ is the power spectrum of $M(t)$. The two components of $P_{I}(f)$ are illustrated in Fig. 1.

The standard theory of white noise shows that when background noise $N$ over bandwidth $B$ is averaged for a time $\tau$, then $\sigma_{N}$, the rms value of $\delta N(t)$, is given by:

$\sigma_{N}=\frac{\langle I\rangle}{\sqrt{B \tau}}$.

$\sigma_{N}$ can also be expressed in the spectral domain:

$\sigma_{N}=\left(\int_{0}^{f_{\text {Nyquist }}} P_{N}(f) d f\right)^{0.5}=\left(<P_{N}>f_{\text {Nyquist }}\right)^{0.5}$.

\footnotetext{
${ }^{1}$ It would be normal to use the terms 'signal power', 'noise power', etc. However, the procedure described refers to the power spectrum of time-variations in the detected signal. The double meaning of the word 'power' might be confusing, so 'power' is only used in connection with the spectrum of time-variation, and other terms used when describing signal output from the receiver square-law detector.
} 
EISCAT IPS 980526 at 93100 UT Source $=0521+166$

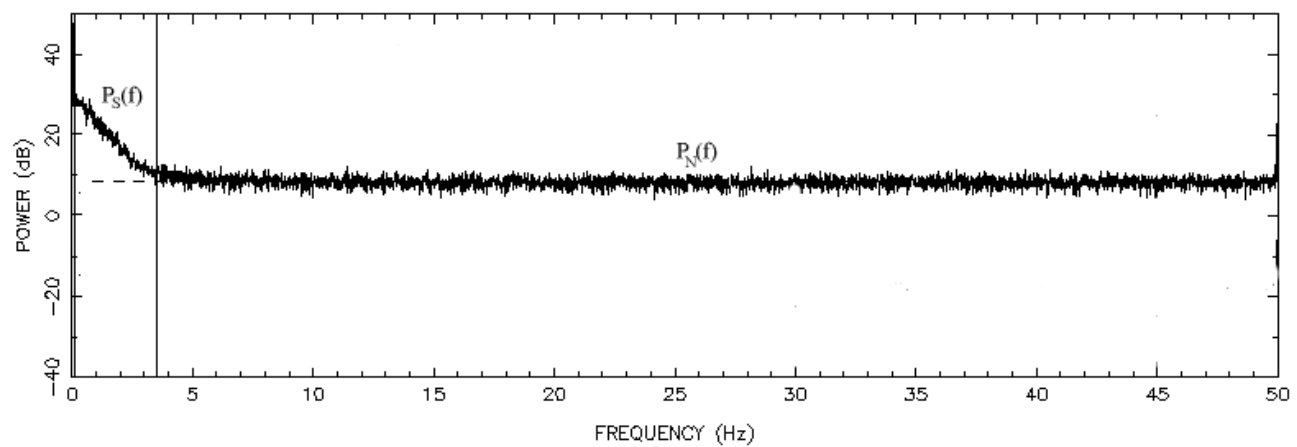

EISCAT IPS 980526 at 93100 UT Source $=0521+166$

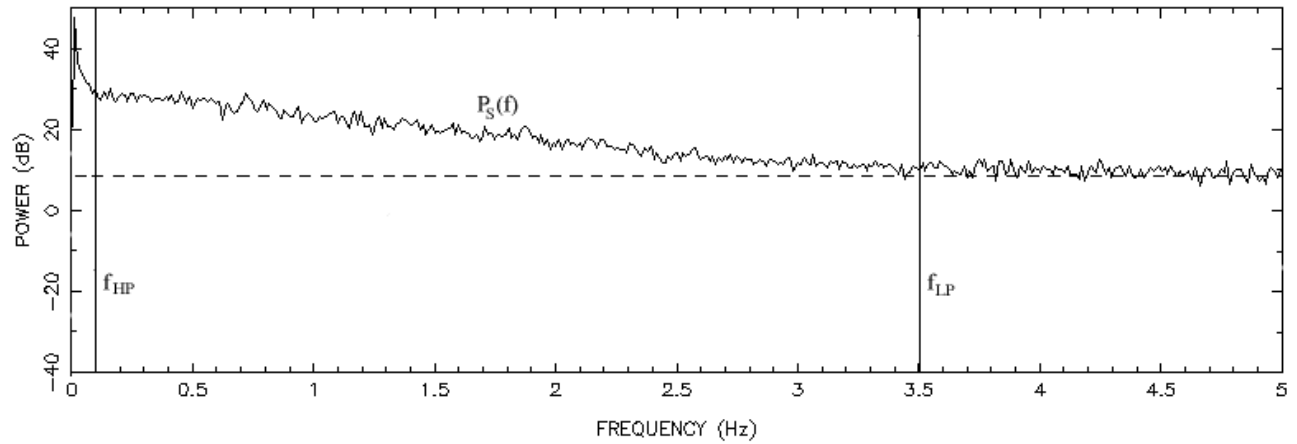

Fig. 1. An example of the power spectrum of the time-variations in detected intensity, calculated from EISCAT IPS measurements. The top panel shows the spectrum out to the Nyquist frequency $(50 \mathrm{~Hz})$. The bottom panel is a close-up of the $0-5 \mathrm{~Hz}$ frequency range, illustrating the position of the low-pass filter $f_{L P}$, which filters out the white spectrum of stochastic noise at higher frequencies, and the high-pass filter $f_{H P}$, which filters out slow time variations within the antenna and receiving systems.

Now $\langle I\rangle=k_{B} T_{\text {sys }} B$ (where $k_{B}=$ Boltzmann's constant; $T_{\text {sys }}=$ system temperature $)$, and the Nyquist frequency is defined by the sampling interval $\tau$ as $f_{\text {Nyquist }}=\frac{1}{2 \tau}$. Hence, substituting Eq. (4) into Eq. (3):

$\left\langle P_{N}\right\rangle=2 k_{B}^{2} T_{\text {sys }}^{2} B$.

Similarly, the rms variation of the scintillating power:

$\sigma_{S c}=\left(\int_{f_{H P}}^{f_{L P}} P_{S c}(f) d f\right)^{0.5}=\left(\left\langle P_{S c}\right\rangle f_{B P}\right)^{0.5}$,

where $f_{B P}$ is the bandwidth $\left(f_{L P}-f_{H P}\right) ;\left\langle P_{S c}\right\rangle$ is the mean of $P_{S c}(f)$ over this bandwidth.

Also:

$\sigma_{S c}=m I_{S}=m k_{B} T_{S} B$,

where $T_{S}$ is the equivalent temperature of the signal from the source.

Hence, by dividing $\sigma_{S c}$ by $\sigma_{N}$, a simple formula for the scintillation index, $m$, can be obtained:

$$
\begin{aligned}
& \frac{\sigma_{S c}}{\sigma_{N}}=\left(\frac{\left\langle P_{S c}\right\rangle f_{B P}}{\left\langle P_{N}\right\rangle f_{\text {Nyquist }}}\right)^{0.5}=\frac{m k_{B} T_{S} B}{k_{B} T_{\text {sys }} \sqrt{2 B f_{\text {Nyquist }}}} \\
& m=\sqrt{\frac{2\left\langle P_{S c}\right\rangle f_{B P} T_{\text {sys }}^{2}}{\left\langle P_{N}\right\rangle B T_{S}^{2}}} .
\end{aligned}
$$

Four of the terms in Eq. (9) are easily measured, or a known constant in the system. However, the system and source temperatures need to be estimated. When a strong radio source passes through the beam of a large antenna, the power from the source itself stands out as a clear, identifiable maximum and the average intensity of the source and the level of scintillation can both be measured directly. For most of the sources used in the present study, however, the signal intensity observed at $931.5 \mathrm{MHz}$ by the $32 \mathrm{~m}$ EISCAT dishes is comparable with the level of "confusion", i.e. the intrinsic variation in background intensity due to stochastic fluctuation in the integrated intensity from all sources, weak and strong, that lie within the antenna beam in any given direction. In such cases, the source intensity cannot be measured directly with sufficient accuracy, and it is necessary to estimate this parameter by using $F_{S}$, the flux density of the source at $931.5 \mathrm{MHz}$

At present, there is no published catalogue of the flux density of radio sources at $931.5 \mathrm{MHz}$, so the flux density is best derived by interpolating accurate measurements - free of confusion - made by more powerful radio telescopes at lower and higher frequencies, and published in the standard catalogues of radio sources. Figure 2 a shows how at frequencies above $400 \mathrm{MHz}$ the flux density of $0521+166$ follows a typical power-law relationship with frequency, so that the 

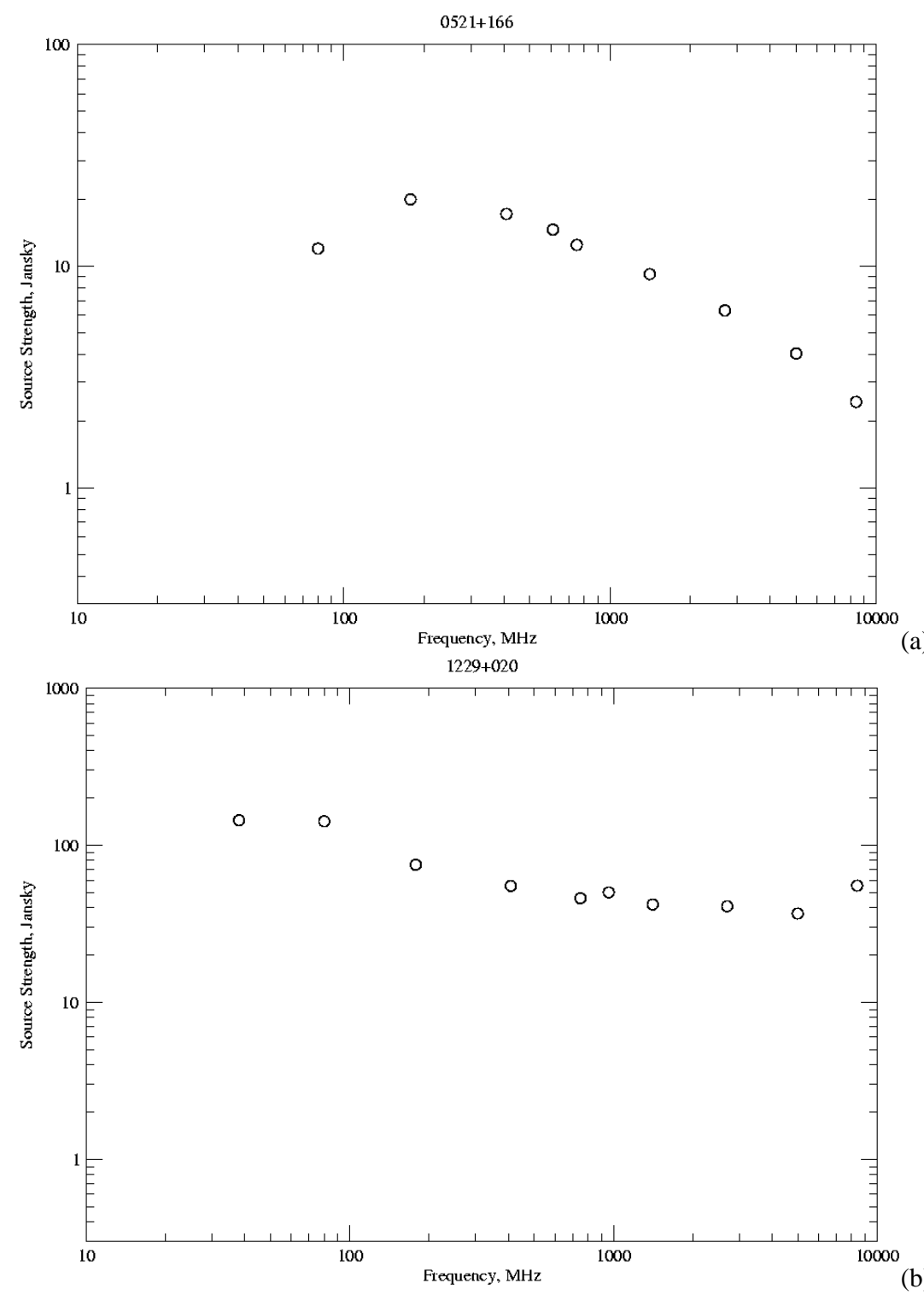

Fig. 2. Log-log plots of $F_{S}$ versus observing frequency for radio sources: (a) $0521+166$, and (b) $1229+020$.

value at $931.5 \mathrm{MHz}$ can be derived by interpolation. The figure also shows the sharp cutoff in flux density at frequencies below $200 \mathrm{MHz}$ : this is the signature of synchrotron self-absorption in radio sources of very small angular diameter (e.g. Williams, 1963; Hornby and Williams, 1966) and hence, a reliable signature of a scintillating source.

Once $F_{S}$ has been estimated, it can be converted into the equivalent $T_{S}$, using $A$, the equivalent collecting area of the EISCAT UHF antennas $\left(=571 \mathrm{~m}^{2}\right.$; Rishbeth and Williams, 1985), and quoting $F_{S}$ in Janskys:

$T_{S}=\frac{F_{S} A}{2 k_{B}}=0.207 F_{S}$.

Even when the total source temperature is determined in this way there is a second problem. The value used to determine $m$ should correspond to the scintillating part of the source, but detailed maps of source intensity (usually made at much higher frequencies) show that the compact scintillating component is often surrounded by a more extended component which is unlikely to scintillate. For example, the strong source $1229+020$ (Fig. 2b) consists of two components, one compact and the other extended. For such a source, the total flux density includes the contribution from the extended, non-scintillating component, and if this is used to calculate the scintillation index, $m$ will always be underestimated. This problem applies to all measurements of $m$ which must, therefore, be regarded as relative and observations of different sources can only be combined after applying some form of calibration to correct for the effects of source structure.

The system temperature can be described by the formula:

$T_{\text {sys }}=T_{\text {receiver }}+T_{\text {ground }}(z)+T_{\text {sky }}+T_{S}$,

where $T_{\text {receiver }}$ is the noise contributed by the receiving system. For Kiruna and Sodankylä, this is mainly due to losses 


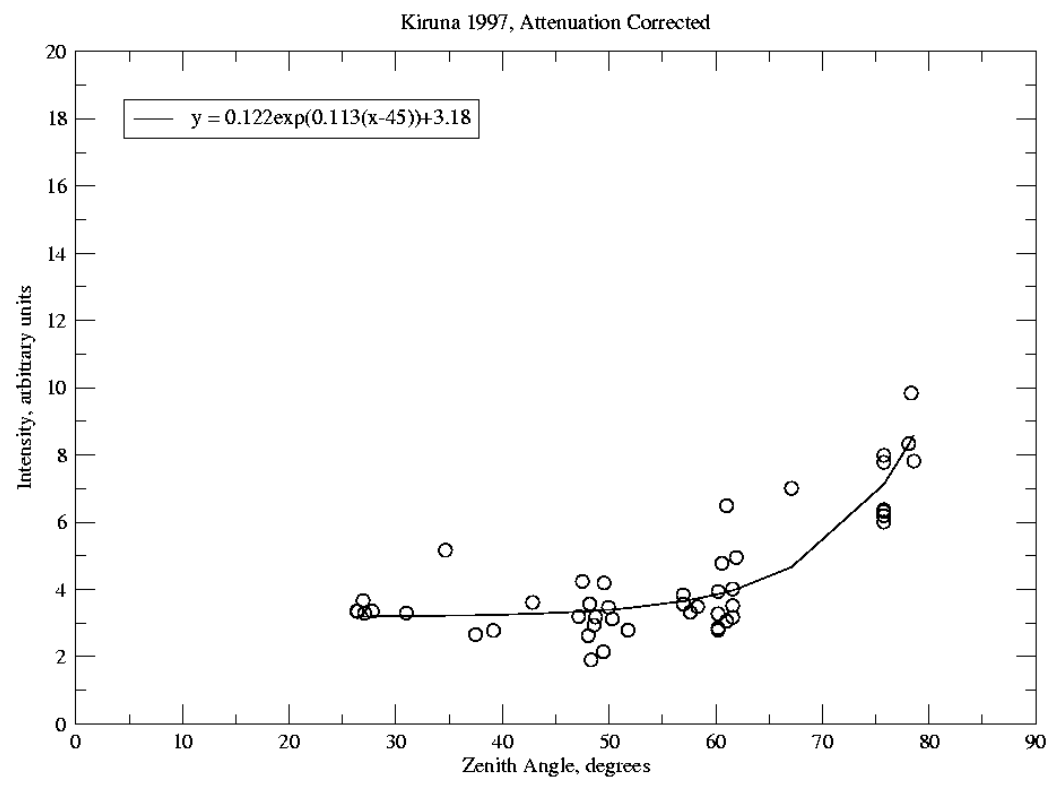

Fig. 3. Example of a plot of detected power versus zenith angle for observations using the antenna at Kiruna in 1997. These data have been corrected for any change in attenuator settings and corrected for the different sources used. The fitted curves are of the form $\left(T_{\text {sys }}-T_{S}\right)=a+b \exp [c(z-45)]$.

before the preamplifier and the preamplifier itself, and is approximately $30 \mathrm{~K}$. At Troms $\varnothing$, the EISCAT transmitting station, there are additional front-end components to protect the receiver from the transmitted signal, so that in the past $T_{\text {receiver }}$ has been approximately $90 \mathrm{~K}$. Data from Troms $\varnothing$ have, therefore, not been used to determine $m$ due to the higher system temperature at that site. (In the future $T_{\text {receiver }}$ will be much lower and it is anticipated that Troms $\varnothing$ will play a greater part in measurements of $m$.)

$T_{\text {ground }}$ corresponds to black-body radiation from the ground coming into the antenna side lobes, and, hence, is a function of zenith angle $z$. $T_{\text {ground }}$ is close to zero for $z<45^{\circ}$, but is significant at lower elevations. (Observations of the powerful radio source Cassiopeia A confirm that for offsets from the main beam $>\sim 45^{\circ}$, the side-lobe levels are always reduced by at least $50 \mathrm{~dB}$ ).

$T_{\text {sky }}$ is the power from the background radiation made up of two isotropic components, the cosmological background and the combined power from radio galaxies and quasars throughout the Universe, and one anisotropic component, the radiation from the Milky Way, which is concentrated near the galactic plane. Hence, $T_{\text {sky }}$ is a function of galactic latitude $b$ and longitude, $l$, but for observations where $b>10^{\circ}$, it can be assumed that $T_{\text {sky }}$ is approximately constant.

Finally, since a specific source is being tracked, the system temperature is systematically higher than the average value by an amount $T_{S}$, dependent on the flux density of the source, as described in Eq. (10). Note that the contribution this makes to the stochastic fluctuation in the background noise has a white-noise power spectrum and is entirely separate from the interplanetary scintillation.

Therefore, for observations where $b>10^{\circ}, T_{\text {sys }}-T_{S}$ should be a simple function of $z$, constant for $z<45^{\circ}$, but increasing with zenith angle for $z>45^{\circ}$. Hence, to estimate the predicted value of $T_{\text {sys }}$ for a particular observation, detected intensity is plotted against zenith angle for each antenna and for each year, using known receiver settings. This monitors any differences between the three antennas and any year-to-year variations in overall receiver performance.

Normally, the measurements follow a fixed relationship between detected power and zenith angle (Fig. 3), determined by fitting the expression:

$\left(T_{\text {sys }}-T_{S}\right)=a+b \exp [c(z-45)]$.

For some measurements, however, the detected power shows a large deviation from the predicted value. This sometimes indicates a fault in the receiver, or a faulty attenuator setting, leading to receiver saturation. On other occasions, it is the result of intermittent interference, usually of human origin. It has been decided, therefore, that the analysis of the scintillation level will only be reliable if the detected power in each case is close to the value predicted by the normal relationship.

To calibrate the observations that follow the normal relationship, a system temperature of $37 \mathrm{~K}$ is assumed for observations at the zenith, and this value is corrected for the effects of ground noise, according to the actual zenith angle of the observation, according to the fitted curve for that antenna and year.

\section{Results}

Once the source and system temperatures are estimated, these figures are put into Eq. (9), to calculate the scintillation indices for each antenna in each observation. The measured values are fewer in number and subject to larger errors than the data derived by systems specifically designed to determine the scintillation index (e.g. the Cambridge system; Duffett-Smith, 1980). However, the ability to estimate 
Table 1.

\begin{tabular}{lccc}
\hline Source: & $\begin{array}{c}\text { Solar Distances } \\
\text { Covered by } \\
\text { Observations } \\
\left(R_{S}\right):\end{array}$ & $\begin{array}{c}\text { Dominant } \\
\text { Stream: }\end{array}$ & $\begin{array}{l}\text { Index } b \text { of } \\
\text { Power-Law } \\
m=a R^{-b}\end{array}$ \\
\hline $0431+206$ & $28-82$ & Slow & $1.65 \pm 0.15$ \\
$0432+416$ & $74-98$ & Fast & $1.72 \pm 0.33$ \\
$0521+166$ & $44-118$ & Slow & $1.84 \pm 0.06$ \\
$0604+203$ & $27-47$ & Slow & $2.08 \pm 0.17$ \\
$0625+146$ & $32-42$ & Fast & $1.38 \pm 0.53$ \\
$0842+185$ & $35-50$ & Slow & $1.45 \pm 0.05$ \\
$1008+075$ & $41-132$ & Slow & $1.58 \pm 0.11$ \\
$1042+120$ & $42-63$ & Slow & $1.97 \pm 0.29$ \\
$1150-003$ & $32-57$ & Slow & $1.81 \pm 0.22$ \\
$1229+020$ & $63-115$ & Slow & $1.52 \pm 0.49$ \\
\hline
\end{tabular}

the distribution of solar wind velocity along the line-of-sight from a single observation, and especially the ability to select those observations where either a fast- or slow-stream accounts for more than $90 \%$ of the scattering, is a major advantage enjoyed by the EISCAT observations. With this capability it is possible for EISCAT to compare the scintillation levels observed when fast- or slow-streams are dominant in the lines-of-sight.

The velocity analysis used requires the use of two antennas. In the case of the EISCAT measurements of the scintillation index, independent values of this can be obtained from either or both of the two stations. In most cases, one of those two stations is at Troms $\varnothing$, where the system noise is much higher than at Kiruna or Sodankylä, so in these cases, only the Kiruna or Sodankylä observations are actually used to provide the scintillation index.

For each source, in turn, the selected measurements of scintillation index are plotted against the closest distance of the line-of-sight from the Sun on a log-log scale (Fig. 4). Slow-stream results are plotted as circles and fast-stream results as triangles. Where appropriate, a least-squares regression is used to fit a simple power-law curve $\left(m \propto R^{-b}\right)$ to the data. In using a least-squares fit, the points were unweighted, since the scatter in the data is greater than $\sigma_{i}$, the estimated standard error in each of the data points (with the exception of $0604+203$ : in this case, the points were weighted by $\left.1 / \sigma_{i}^{2}\right)$.

The values of $m$ are relative and not absolute. The error introduced by using the whole flux density of the source, and not just the scintillating component, will systematically underestimate all calculated values of $m$, but to the same extent. Errors in the estimate of $F_{S}$, and in the assumed system temperature, will also affect all observations of a given source in more-or-less the same way, though for these errors $m$ may be systematically underestimated or overestimated. Finally, there are random errors in the relative values, and these have
Table 2.

\begin{tabular}{lccc}
\hline Paper: & $\begin{array}{c}\text { Source } \\
\text { (or average } \\
\text { over } \\
\text { sources): }\end{array}$ & $\begin{array}{c}\text { Solar } \\
\text { Distances } \\
\left(R_{S}\right) \text { : }\end{array}$ & Exponent, $b$ : \\
\hline $\begin{array}{l}\text { Bourgois, 1969 } \\
\text { Armstrong and }\end{array}$ & $\begin{array}{c}\text { Average } \\
\text { Crab pulsar }\end{array}$ & $10-90$ & $1.55 \pm 0.05$ \\
Coles, 1978 & & & $1.55 \pm 0.15$ \\
Manoharan, 1993 & $1256-057$ & $80-200$ & $1.89 \pm 0.15$ \\
& $1058+015$ & $80-200$ & $1.72 \pm 0.20$ \\
& $1229+020$ & $75-200$ & $1.78 \pm 0.16$ \\
& $1008+075$ & $75-200$ & $1.59 \pm 0.20$ \\
Coles et al., 1995 & $1256-057$ & $5-15$ & $1.70 \pm ?$ \\
Asai et al., 1998 & $1058+015$ & $50-215$ & $1.60 \pm ?$ \\
\hline
\end{tabular}

been estimated by measuring $m$ separately for each 3-min segment of the usual 15-min observation. This gives five independent indices for each observation, and these are used to determine a mean and standard error. These standard errors are also given in Fig. 4.

\subsection{Results for individual sources}

Figure 4a shows all the results for the source $0431+206$ where a single mode is dominant. In this case, there are 5 slow-stream measurements of $m$ at distances ranging from 28 to $82 R_{S}$. These follow closely the simple power-law relationship $m \propto R^{-b}$, with the index $b=1.65 \pm 0.15$. There are no fast-stream measurements for this source.

In this respect, $0432+416$ (Fig. 4b) is complementary. Here, there are only 3 slow-stream observations and 5 faststream. All the data are confined within the distance range 74 to $98 R_{S}$, so that neither can be used to determine an accurate power-law relationship between $m$ and $R$, though the faststream data give $b=1.72 \pm 0.33$. However, a comparison between the two sets of data indicates that at a distance of $\sim 80 R_{S}$, the value of $m$ for the fast-stream is a factor of $2.2 \pm 0.1$ times smaller than for the slow-stream.

$0521+166$ (Fig. 4c) shows a power-law relationship between $m$ and $R$ for slow-stream measurements, and the difference in scintillation index between slow- and fast-streams. Four measurements of $m$ in the slow-stream, ranging from 44 to $118 R_{S}$, suggest a power-law relationship with an index of $1.84 \pm 0.06$ : it must be noted, however, that this value depends heavily on a single value at $118 R_{S}$. There is also a single fast-stream observation at a distance of $29 R_{S}$ and this time $m$ is a factor of $2.4 \pm 0.1$ lower than the value obtained for this distance by extrapolation from the slow-stream data.

Figures $4 \mathrm{~d}-\mathrm{j}$ give the results obtained using 7 other sources. By and large, they confirm the results derived from Figs. 4a-c. Thus, 0842,185 (Fig. 4f), $1008+075$ (Fig. 4g), 1150-003 (Fig. 4i) and $1229+020$ (Fig. 4j) are similar to $0431+206$ (Fig. 4a), and $0521+166$ (Fig. 4c), with slow- 

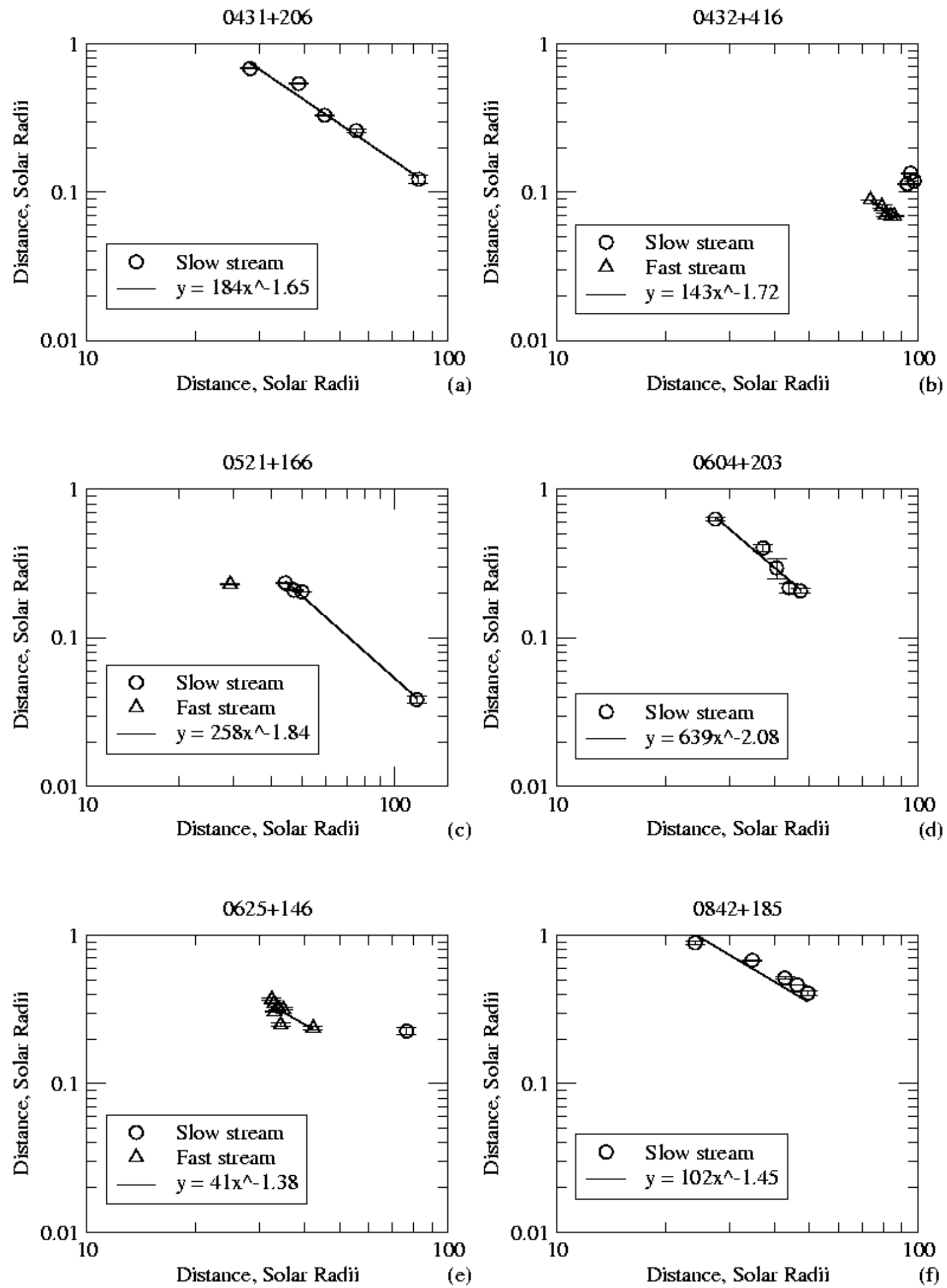

Fig. 4. Log-log plots of relative scintillation index versus distance from the Sun for a number of sources and cases where a single fast- or slow-stream is dominant across the line-of-sight. The curves fitted are of the form $y=a x^{-b}$. Errors in the $y$-values have been plotted. See text for details. 
$1008+075$

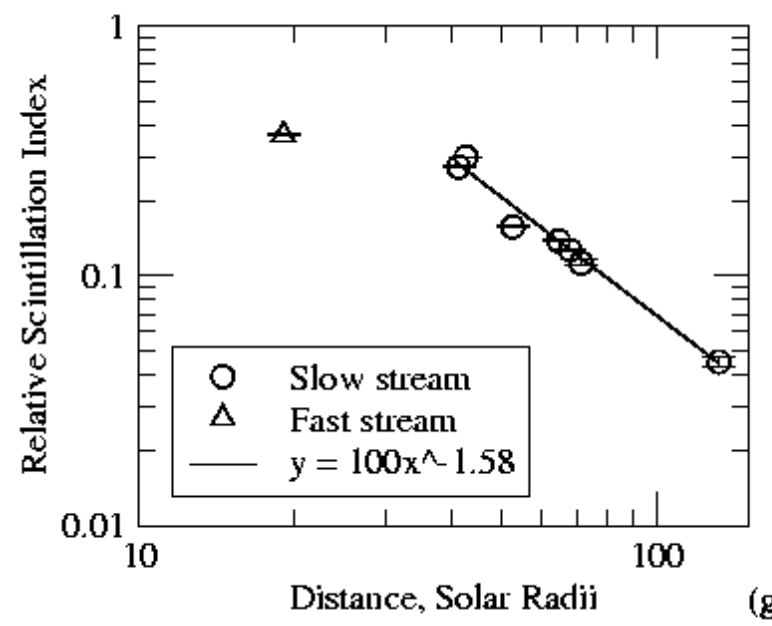

$1150-003$

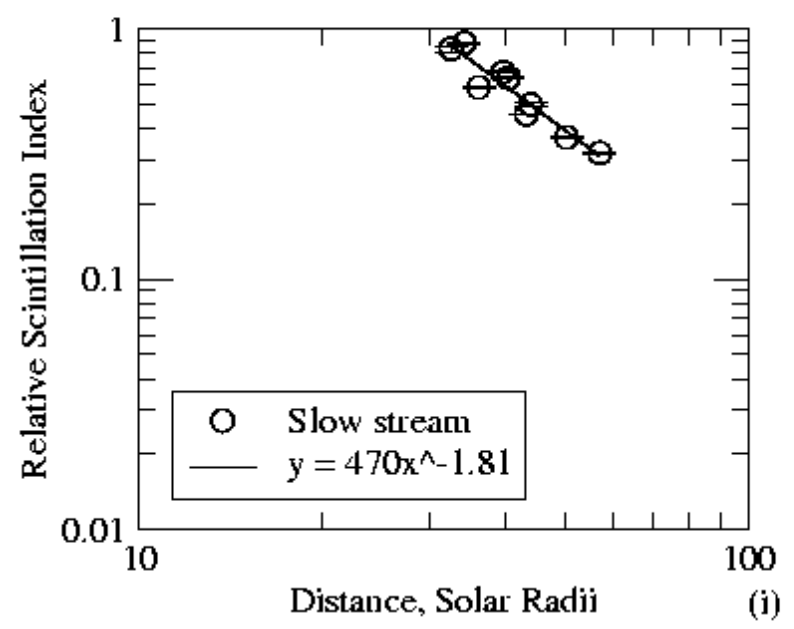

$1042+120$



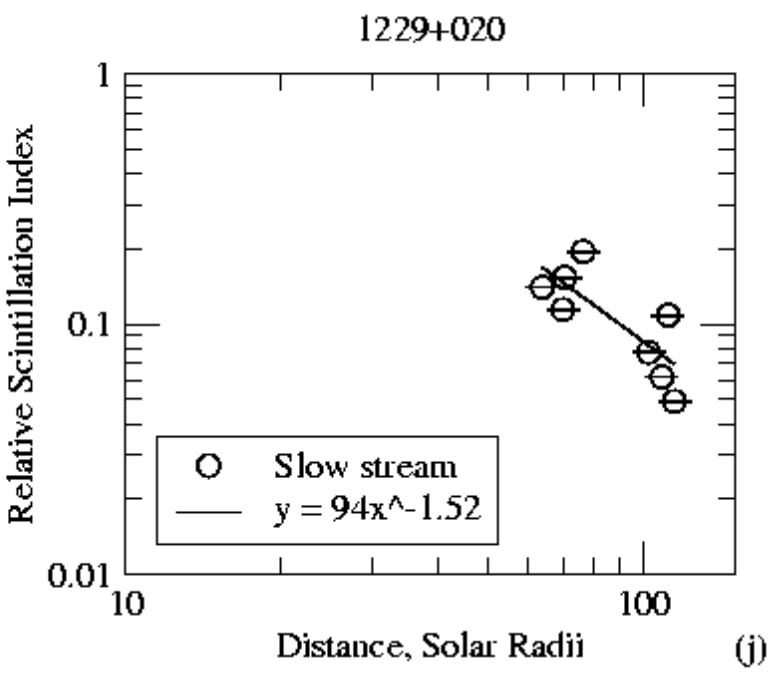

Fig. 4. continued ...

stream measurements between them covering 24-132 $R_{S}$. All six cases show a similar power-law dependence, with values of $b$ lying in the range $1.45 \pm 0.05$ to $1.84 \pm 0.06$.

Thus, for $1008+075$ (Fig. 4g), there are 7 slow-stream observations, ranging from 41 to $132 R_{S}$, and they show a well-defined power-law relationship between $m$ and $R$, with an index $b$ of $1.58 \pm 0.11$. There is also a single fast-stream observation at $R=19 R_{S}$. In this case, the measured value of $m$ is a factor of $2.6 \pm 0.3$ lower than the value extrapolated from the slow-stream observations. This could be a combination of the lower values of $m$ always found in the fast-stream, plus the transition from weak- to strong-scattering, which is likely in the fast-stream at $19 R_{S}$.

In contrast, it should be noted that in two cases where only slow-stream observations are available, $0604+203$ (Fig. 4d), and $1042+120$ (Fig. $4 \mathrm{~h}$ ), there is apparently a faster decrease in $m$ with increasing $R$, and in both cases, $b$ is $\sim 2.0$.

Finally, $0625+146$ (Fig. 4e) is unusual because in this case, there are 7 fast-stream observations and only one slowstream. All the fast-stream observations are within the range 32 to $42 R_{S}$ and the values of $m$ show considerable scatter, so that although the measurements are consistent with a powerlaw relationship between $m$ and $R$, only an estimate of the index $b$ can be made $(1.38 \pm 0.53)$. However, when this relationship is extrapolated for comparison with the single slowstream measurement at $76 R_{S}$, the ratio of $2.2 \pm 1.0$ is at least consistent with the other measurements.

\subsection{Overall results}

The expected power-law was observed throughout the weak scattering regime over the range of distances covered by these measurements, and curves of the form $m=a R^{-b}$ were fitted in all cases where adequate data were available.

The exact form of the power law varies from $R^{-1.38}$ to $R^{-2.08}$ (Table 1), and this gives a weighted mean of $1.64 \pm$ 0.04 . (This includes both fast- and slow-stream cases, since 




Fig. 5. Normalised log-log plots of relative scintillation index versus distance from the Sun for cases where a single fast- or slow-stream is dominant across the line-of-sight. The curves fitted are of the form $y=a x^{-b}$.

no significant difference can be seen in the power law for either category).

To derive the best overall results, it is necessary to combine the data from all 10 sources. The main difficulty in doing this is that all the individual measurements of $m$ are relative values: each of them is likely to be systematically underestimated but to different extents. However, in 8 of the 10 cases, the slow-stream measurements of $m$ are seen to follow a simple power-law relationship with distance, and in 6 cases, the range of distances actually covered by the slow-stream measurements includes $50 R_{S}$. Therefore, all the slow-stream data sets, where it was possible to fit a power law, were normalised at $50 R_{S}$, to give a revised value of $m=0.30$. Figure 5 shows the plot of the normalised values of $m$ versus $R$ and the best-fitting power-law relationship, $m \propto R^{-1.64}$.

In only two cases was it possible to fit separate powerlaw relationships to the fast-stream data. However, on the assumption that the normalising factor compensates for any error in the estimate of $F_{S}$, and for the extent to which the scintillating component of $F_{S}$ was significantly less than $F_{S}$, it should be the same for both slow- and fast-streams. For any given source, therefore, the same normalisation factor was applied to the fast-stream data as had been calculated for the slow-stream data. Figure 5 shows the plot of the normalised fast-stream values of $m$ versus $R$. Once again, the best-fitting power-law relationship is given by $m \propto R^{-1.64}$. In other words, the ratio of the slow-stream scintillation index to the fast-stream index equals 2.3 at all solar distances from 30 to $90 R_{S}$.

As measurements in the weak scattering regime are made closer and closer to the Sun, the scintillation index rises to a peak, but even closer the line-of-sight passes through a strong scattering regime and the scintillation index is ex- pected to drop rapidly. At the EISCAT observing frequency of $931.5 \mathrm{MHz}$, this peak is expected to occur at about 25 $30 R_{S}$ for the slow-stream and about $15-20 R_{S}$ for the faststream. Very few data are available within a solar distance of $30 R_{S}$, but Fig. 5 suggests that the transition from weakto strong-scattering is close to $24 R_{S}$ for the slow-stream and below $19 R_{S}$ for the fast-stream. (There are measurements of the scintillation index close to the Sun that show the drop in $m$ inside the strong scattering regime, but all of these data have been excluded from the present paper, because in no case does either the fast- or slow-stream contribute more than $90 \%$ of the total scattering.)

\section{Discussion and conclusions}

The scintillation indices calculated above are relative values, since in order to obtain an absolute value, it would be necessary to know the detailed structure of each source at the observing frequency of $931.5 \mathrm{MHz}$, and then correct for the contribution to the overall source temperature from nonscintillating components. The effect of source structure on the results is demonstrated by the variation in the overall level of scintillation in the results from different individual sources.

Nevertheless, the variation of the scintillation index with distance from the Sun is clearly seen. It was expected that a peak in the scintillation index would be visible, indicating the transition between strong- and weak-scattering regimes. However, with few totally reliable measurements available within $30 R_{S}$, a decrease in the scintillation index within the strong-scattering regime is not seen in any of the data where one stream is more than $90 \%$ dominant. The only hint visible is a flattening of the scintillation index curve in the measure- 
ments of $0842+185$ (Fig. 4f) for the slow-stream and 1008 + 075 (Fig. 4g) for the fast-stream. However, measurements close to the Sun, where the slow-stream is dominant - but not 90\% dominant - do suggest a sharp reduction in the scintillation index inside $20 R_{S}$.

Beyond $30 R_{S}$, within the weak-scattering regime for observations at this frequency, the scintillation index decreases with distance from the Sun as a simple power law. The weighted mean exponent of this power law is found to be $1.64 \pm 0.04$. Comparison of this value with that previously published by other authors (e.g. Bourgois, 1969; Armstrong and Coles, 1978; Coles et al., 1995; Asai et al., 1998) shows a good agreement (Table 2).

These values are close to the index expected from simple theory. Within the weak scattering regime, the contributions to the overall variation in intensity $(\delta I)$ from different parts of the line-of-sight are independent. Therefore, the variance of these contributions $\left(\delta I^{2}\right)$ adds. Hence, assuming that $\delta I \propto \delta N_{e}$ (the rms variation in the density) $\propto R^{-2}$, it follows that $\delta I^{2} \propto R^{-4}$. However, the path length of the line-of-sight is proportional to $R$. Therefore, the path integral of $\delta I^{2}$ is proportional to $R^{-3}$, and the rms variation in the measured intensity of the source $\propto R^{-1.5}$ (Coles et al., 1995). The average index in the measured power law is, therefore, slightly greater than predicted by this simple theory, indicating that the rms variation in the density follows a slightly steeper power law than an inverse square. This is indeed to be expected. If solar wind velocity increases as $R^{\alpha}$, then in a steady state the electron density actually falls with distance as $R^{-(2+\alpha)}$. The scintillation index should, therefore, be proportional to $R^{-(1.5+\alpha)}$ and $b=(1.5+\alpha)$. Model results of the fast solar wind from Allen et al. (2000) indicate that $0.05<\alpha<0.1$ over the distance range $30-100 R_{S}$. A similar model for the slow-stream (Chen and $\mathrm{Hu}, 2001$ ) indicates that $\alpha \sim 0.1$.

However, in individual cases, the power laws varied from $R^{-1.38}$ to $R^{-2.08}$. Similar variations of the power law from source to source were also seen by Manoharan (1993), using observations over a distance range of $80 R_{S}$ to $200 R_{S}$. The sources $1008+075$ and $1229+020$ formed part of this author's study and the exponents he published are in agreement with those presented here for the same sources, within the bounds of error.

A difference between the levels of scintillation due to fastand slow-streams across the line-of-sight is evident in the limited data available. The main example is seen in the observations of $0432+416$. Here, the relative scintillation indices when the line-of-sight is entirely within the slow-stream are found to be 2.2 times greater than when it is within the fast stream. When measurements from different sources are combined (Fig 5), the ratio of the slow-stream scintillation index to that of the fast-stream is 2.3 over the distance range 30 to $90 R_{S}$.

Measurements of the same source which feature both dominant fast-streams and dominant slow-streams are naturally limited. For a dominant fast-stream to be measured, the source needs to have a high heliographic latitude and to be measured at solar minimum (when slow flow in the ends of the line-of-sight is minimised); whereas for a dominant slowstream to be measured, it needs to be at low latitude or to be measured at solar maximum. These limitations, combined with data quality controls, are the reasons for the limited number of dominant fast-stream data. However, despite these limitations, the difference seen is in good agreement with previous studies outside of the acceleration region (Manoharan, 1993; Asai et al., 1998).

Acknowledgements. We would like to thank the director and staff of EISCAT for the data used in this study. EISCAT is supported by the scientific research councils of Finland, France, Germany, Japan, Norway, Sweden, and the UK. Two of us (RAF and ARB) were supported by PPARC during the period when this work was carried out. We would like to give particular thanks to W. A. Coles for his help in the derivation of the scintillation index.

Topical Editor M. Lester thanks M. Kojima and another referee for their help in evaluating this paper.

\section{References}

Allen, L., Habbal, S., and Li, X.: Thermal coupling of protons and neutral hydrogen with anisotropic temperatures in the fast solar wind, J. Geophys. Res., 105, 23 123-23 134, 2000.

Armstrong, J. and Coles, W.: Interplanetary scintillations of psr 0531+21 at $74 \mathrm{mhz}$, Astrophys. J., 220, 346-352, 1978.

Asai, K., Kojima, M., Tokumaru, M., Yokobe, A., Jackson, B., Hick, P., and Manoharan, P.: Heliospheric tomography using interplanetary scintillation observations 3. correlation between speed and electron density fluctuations in the solar wind, J. Geophys. Res., 103, 1991-2001, 1998.

Bourgois, G.: Scintillations interplanétaires des radiosources à 2695mhz, Astron. Astrophys, 2, 209-217, 1969.

Bourgois, G. and Coles, W.: Solar cycle changes in the turbulence level of the polar stream near the sun, in: Solar Wind Seven, (Eds) Marsch, E. and Schwenn, R., pp. 155-158, Pergamon, New York, 1992.

Bourgois, G., Coles, W., Daigne, G., Silen, J., Turunen, T., and Williams, P.: Measurements of the solar wind velocity with eiscat, Astron. Astrophys., 144, 452-462, 1985.

Breen, A., Coles, W., Grall, R., Klinglesmith, M., Markkanen, J., Moran, P., Tegid, B., and Williams, P.: Eiscat measurements of the solar wind, Ann. Geophysicae, 14, 1235-1245, 1996 b.

Breen, A., Moran, P., Varley, C., Wilkinson, W., Williams, P., Coles, W., Lecinski, A., and Markkanen, J.: Interplanetary scintillation observations of interaction regions in the solar wind, Ann. Geophysicae, 16, 1265-1282, 1998.

Briggs, B.: The correlation of radio star scintillations with geomagnetic disturbances, Geophys. J., 5, 306-317, 1961.

Canals, A., Breen, A., Moran, P., and Ofman, L.: Estimating random transverse velocities in the fast solar wind from eiscat interplanetary scintillation measurements, Ann. Geophysicae, this issue, 2002.

Chen, Y. and Hu, Y.: A two-dimensional Alfvén-wave-driven solar wind model, Sol. Phys., 199, 371-384, 2001.

Coles, W.: A bimodal model of the solar wind speed, Astrophys. Space Sci., 243, 87-96, 1996.

Coles, W., Grall, R., Klinglesmith, M., and Bourgois, G.: Solar cycle changes in the level of compressive microturbulence near the sun, J. Geophys. Res., 100, 17 069-17 079, 1995. 
Dennison, P. and Hewish, A.: The solar wind outside the plane of the ecliptic, Nature, 213, 343-346, 1967.

Duffett-Smith, P.: The measurement of interplanetary scintillations in conditions of strong radio interference, Mon. Not. R. Astr. Soc., 190, 139-141, 1980.

Gapper, G., Hewish, A., Purvis, A., and Duffett-Smith, P.: Observing interplanetary disturbances from the ground, Nature, 296, 633-636, 1982.

Grall, R.: Remote Sensing Observations of the Solar Wind Near the Sun, Ph.D. thesis, University of California, San Diego, 1995.

Grall, R., Coles, W., Klinglesmith, M., Breen, A., Williams, P., Markkanen, J., and Esser, R.: Rapid acceleration of the polar solar wind, Nature, 379, 429-432, 1996.

Hewish, A., Scott, P., and Wills, D.: Interplanetary scintillation of small diameter radio sources, Nature, 203, 1214-1217, 1964.

Hornby, J. and Williams, P.: Radio sources having spectra with a low frequency cut-off, Mon. Not. R. Astr. Soc., 131, 237-246, 1966.

Jackson, B., Hick, P., Kojima, M., and Yokobe, A.: Heliospheric tomography using interplanetary scintillation observations: 1 . combined nagoya and cambridge data, J. Geophys. Res., 103, 12 049-12 067, 1998.

Klinglesmith, M.: The Polar Solar Wind from 2.5 to 40 Solar Radii: Results of Intensity Scintillation Measurements, Ph.D. thesis, University of California, San Diego, 1997.

Kojima, M., Tokumaru, M., Watanabe, H., Yokobe, A., Asai, K., Jackson, B., and Hick, P.: Heliospheric tomography using interplanetary scintillation observations: 2 . latitude and heliocentric distance dependence of solar wind structure at 0.1-1 au, J. Geophys. Res., 103, 1981-1989, 1998.

Little, L. and Hewish, A.: Interplanetary scintillation and its relation to the angular structure of radio sources, Mon. Not. R. Astr. Soc., 134, 221-237, 1966.

Manoharan, P.: Three-dimensional structure of the solar wind: Variation of density with the solar cycle, Sol. Phys., 148, 153-167, 1993.

Manoharan, P. and Ananthakrishnan, S.: Determination of solar wind velocities using single-station measurements of interplanetary scintillation, Mon. Not. R. Astr. Soc., 244, 690-695, 1990.

Massey, W.: Measuring intensity scintillations at the very long baseline array (vlba) to probe the solar wind near the sun, M.Phil. thesis, 1998.

Moran, P., Ananthakrishnan, S., Balasubramanian, V., Breen, A., Canals, A., Fallows, R., Janardhan, P., Tokumaru, M., and Williams, P.: Observations of interplanetary scintillation during the 1998 whole sun month: a comparison between eiscat, ort and nagoya data, Ann. Geophysicae, 18, 1003-1008, 2000.

Phillips, J., Bame, S., Feldman, W., Goldstein, B., Gosling, J., Hammond, C., McComas, D., Neugebauer, M., Scime, E., and Suess, S.: Ulysses solar wind plasma observations at high southerly latitudes, Science, 268, 1030-1033, 1995.

Rishbeth, H. and Williams, P.: The eiscat ionospheric radar: the system and its early results, Q. J. R. Astr. Soc., 26, 478-512, 1985.

Schwenn, R., Montgomery, M., Rosenbauer, H., and Miggenrider, H.: Direct observations of the latitudinal extent of a high-speed stream in the solar wind, J. Geophys. Res., 83, 1011-1017, 1978.

Vitkevich, V. and Vlasov, V.: Radioastronomical investigations of the drift of the inhomogeneous interplanetary plasma, Sov. Astron. - AJ, 13, 669-676, 1970.

Williams, P.: Absorption in radio sources of high brightness temperature, Nature, 200, 56-57, 1963. 